\title{
IMPLEMENTATION OF AN AUTONOMOUS REACTIVITY CONTROL SYSTEM IN A SMALL LEAD-COOLED FAST REACTOR
}

\author{
Fredrik Dehlin, Govatsa Acharya, Sara Bortot and Ignas Mickus \\ KTH, Division of Nuclear Engineering \\ Albanova University Center, 10691 Stockholm, Sweden \\ fdehlin@kth.se, govatsa@kth.se, bortot@kth.se, mickus@kth.se
}

\begin{abstract}
This paper describes the design, implementation and characterisation of an Autonomous Reactivity Control (ARC) system in a small modular lead-cooled fast reactor. The aim of this work was to demonstrate the applicability of the ARC system and to study its dynamic behaviour during an anticipated transient without scram. A simplified one-dimensional model was developed to calculate the heat transfer within the ARC system, and the reactivity worth as a function of the neutron poison's insertion into the active core was obtained via static neutronic calculations. By coupling the aforementioned models, the ARC's time-dependent reactivity was derived as a function of the coolant outlet temperature variation. This model was implemented into the BELLA multi-point dynamics code and transient simulations were run. A control rod ejection accident was studied leading to an unprotected transient overpower scenario, in which $350 \mathrm{pcm}$ reactivity was inserted during one second. It was shown that the ARC system provides a forceful negative reactivity feedback and that steady-state temperatures after the transient were reduced by almost $300 \mathrm{~K}$ compared to an identical transient without its action. In this scenario, the ARC system managed to stabilise the coolant outlet temperature at a value $100 \mathrm{~K}$ above nominal conditions. The implementation of an ARC system provided the reactor with a passively actuated self-regulating reactivity control system able to insert large amounts of negative reactivity in a short amount of time.
\end{abstract}

KEYWORDS: Autonomous reactivity control, small modular reactor, lead-cooled fast reactor, passively actuated safety systems

\section{INTRODUCTION}

In one of the more recent major reports published by the Intergovernmental Panel on Climate Change (IPCC), SPECIAL REPORT: Global Warming of $1.5^{\circ} \mathrm{C}$ [1], nuclear power is modelled to increase its total capacity with between $98 \%$ up to $501 \%$ in the year 2050, compared to the installed capacity in 2010. To achieve this ambitious target, many of the obstacles currently facing large scale nuclear power construction projects, e.g. cost overruns or increased safety requirements, needs to be overcome. In this paper we study the implementation of an innovative passive selfactuated reactivity control system installed in a state-of-the-art small lead-cooled fast reactor. The main objective of this safety system is to passively respond to and mitigate unprotected transient scenarios. Moreover, implementation of a passive safety system will increase the overall safety 
and consequentially increase the general public's acceptance of nuclear technologies, which is of utmost importance if the ambitious goals set out in the Paris Climate Accord is to be achieved.

In this paper the Autonomous Reactivity Control (ARC) system [2-4], developed by Dr Staffan Qvist, is implemented into the small modular lead-cooled fast reactor (LFR) SEALER-UK [5], and its behaviour is assessed during an Anticipated Transient Without SCRAM. The ARC system has previously been implemented and analysed to various degree in different sodium fast reactor designs [2-4,6,7], however, this work marks the first time that the ARC system has been implemented in a lead-cooled reactor. First order solution methods are employed to study the dynamic behaviour of the reactor with and without the passive safety system installed.

\section{AUTONOMOUS REACTIVITY CONTROL}

\subsection{SEALER-UK}

SEALER-UK or the Small, Economic and Agile Lead-Cooled Reactor for the United Kingdom is a small modular lead-cooled reactor developed by the Stockholm based company LeadCold Reactors AB. The reactor is a redesigned and upscaled version of the SEALER-Arctic [8] reactor, and it is designed to deliver $55 \mathrm{MWe}$. One aspect that differentiates this design from other LFRs is the use of advanced FeCrAl-alloys [9] to overcome issues with corrosion normally associated with the use of high temperature liquid lead. As fuel, SEALER uses $11.8 \%$ enriched uranium nitride (UN) placed in 85 fuel assemblies. Reactivity control is mainly performed with six boron carbide $\left(\mathrm{B}_{4} \mathrm{C}\right)$ control rod assemblies along with six wolfram-rhenium diboride $\left(\mathrm{W}-(\mathrm{W}, \mathrm{Re}){ }^{10} \mathrm{~B}_{2}\right)$ shut down assemblies located in the periphery of the core.

The SEALER-UK reactor is designed to be operated in a breeder/ burner configuration, in which it breeds until middle of life and subsequently burns until end of life, with a total fuel cycle length of 22.5 years. Thereafter the reactor is intended to be taken offline and either sent for fuel reprocessing or to final long-term storage.

\subsection{ARC Design}

The ARC system is designed to be a simple and cost-effective addition to greatly enhance reactor safety. To reduce the impact on both fuel assembly manufacturing and on core design, the ARC system is made to fit the existing fuel assembly designs with slight modifications. The ARC can, in short, be explained as a spring-loaded passive self-regulating system that autonomously inserts neutron poison into the core when exposed to an increased coolant outlet temperature

Fundamentally the ARC system is made up of three main parts, as seen in Figure 1 hereinunder. The two reservoirs, the upper and the lower, are respectively located on the coolant outlet tube and the coolant inlet tube, and they are connected via two concentric tubes that allow fluid to flow between them. The outer tube is made to the same dimensions as a fuel rod and it will thus simply replace the fuel rod in the fuel assembly without any extra modification. The ARC system is filled with two liquids and one gas, and as seen in Figure 1 the upper reservoir is divided into two compartments. The uppermost of the two compartments is filled with the liquid potassium, 
hereinafter known as the expansion liquid, and it has the primary task to expand volumetrically as a response to an increase of the coolant outlet temperature.

From the upper compartment the expansion liquid is connected to the lower reservoir via the innermost of the two concentric tubes. This enables the volumetric expansion to propagate down into the lower reservoir, which is partly filled with expansion fluid. Floating on top of the expansion fluid is an immiscible neutron poison, proposed to be liquid lithium with a high ${ }^{6} \mathrm{Li}$ enrichment, and hereinafter known as the absorber liquid. The remaining volume of the lower reservoir, the volume between the two concentric tubes and the volume of the lower compartment in the upper reservoir is occupied by an inert gas. This gas is proposed to be argon and it has the primary task to act with an opposing force on the liquids, thus forcing the poison out of the core once the transient has dissipated and the coolant outlet temperature has decreased.

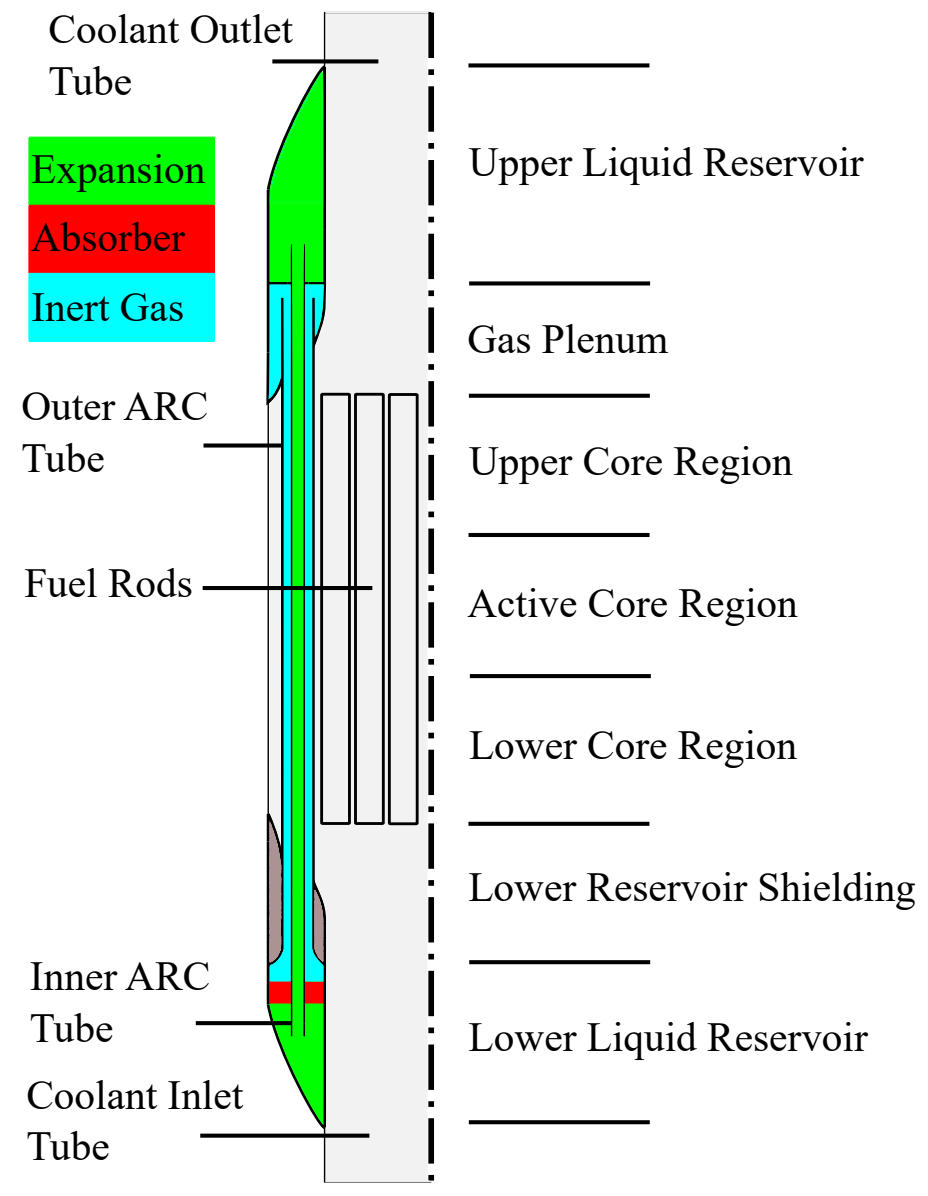

Figure 1: Main components of the ARC system installed on a fuel assembly.

A significant portion of the design choices made are tailored to the required reactivity response, which in turn depends on the reactor design in question and its expected worst-case transient scenario. The amount of negative reactivity inserted into the core by the ARC system depends mainly on two factors: the ${ }^{6} \mathrm{Li}$ enrichment and the volume fraction of absorber liquid in the core. An example of what the ARC system might look like when it is implemented in a generic LFR fuel 
assembly is seen in Figure 2. The designer can choose different layouts and ratios between the inner and the outer ARC tubes in order to tailor the reactivity response to one particular reactor design.

Before implementing a final version of the ARC system into a reactor one has to optimise the dynamic response of the ARC system by varying the ratio between the radius of the outer and the inner ARC tubes. A larger ratio would imply that more absorber liquid is forced into the core, however with the consequence that the actuation time is slower, and the contrary is true for a design utilising a smaller ratio. In some reactor designs it might be more important with a quick actuation of a small amount of reactivity, whilst in others, the opposite might be true.

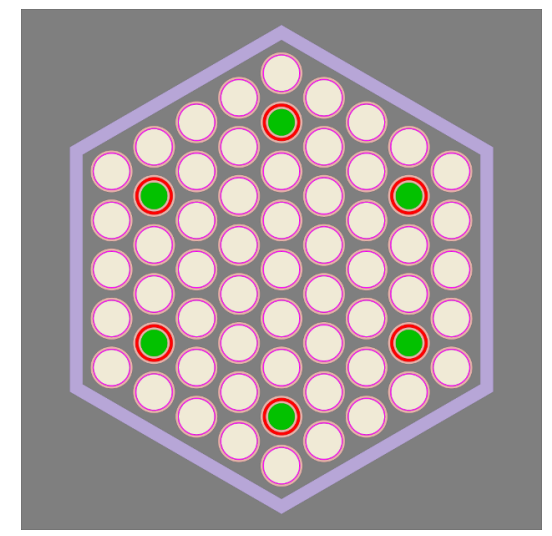

Figure 2: Generic LFR fuel assembly with six ARC tubes implemented. Note: Not to scale.

For the implementation of the ARC system into the SEALER-UK reactor it was decided to replace six fuel rods with ARC tubes and that the system should be able to insert, the very least, $0.5 \$$ of reactivity $\left(\Delta \rho_{A R C} \geq 289 \mathrm{pcm}\right)$. For additional safety, it was however decided that in this preliminary analysis the ARC system should be able to insert $\Delta \rho_{A R C}=350 \mathrm{pcm}$. It was also decided to use a uniform tube layout, i.e. the ratio between the radius of the outer ARC tube and the inner ARC tube is kept constant in every fuel assembly, thus obtaining a symmetric response to a temperature increase. For future studies it could be interesting to optimise the tube ratios depending on their spatial location within the core, e.g. a larger ratio in the periphery compared to that in the centre.

\subsection{ARC Modelling and BELLA Implementation}

BELLA, or Bortot's Elegant Liquid LFR Analysis tool [10-14] is a multi-point dynamics code that incorporates a lumped parameter approach, in which a point wise description of, and coupling between, the reactor core, primary coolant and steam generators are utilised. BELLA was created to enable a preliminary safety informed design of metal cooled fast reactors without the need for complex and sometimes expensive proprietary software.

A model of the ARC system was developed and implemented in the BELLA tool together with a model of the SEALER-UK reactor. The model of the ARC system can be divided into three main parts; the first part deals with the heat transfer from the coolant outlet tube into the upper reservoir, 
the second part determines a correlation between the inserted height of absorber liquid into the core as a function of expansion liquid temperature and the third part correlates the inserted height of absorber liquid with the amount of inserted negative reactivity.

\subsubsection{Heat transfer in upper reservoir}

A wide variety of methods to model heat transfer exist, ranging from simpler one-dimensional approaches to advanced multi-physics simulations. For this work it was decided to use a simplified fist order heat transfer model based around the one-dimensional heat equation and to solve it with a second order central finite difference method. It was assumed that heat was transferred both from the coolant flowing in the coolant outlet tube and from the coolant in the bypass flow by convective heat transfer. Inside the system, in order to simplify the model, it was assumed that conductive heat transfer was the only heat transfer process taking place, whilst in reality there would be a mix between conductive and convective heat transfer in the liquid parts of the system.

The finite difference scheme allows for the construction of a matrix equation which is solved by the BELLA software to obtain a one-dimensional temperature distribution between the two known boundaries, i.e. the coolant outlet temperature and the bypass flow temperature. At every point in time an arithmetic mean value is calculated based on the temperature distribution in the expansion liquid, and this value is passed on to the next segment of the ARC solver. The omission of convective heat transfer does however reduce the physical accuracy of the model, and it also increases the response time of the ARC system to a change in temperature. This assumption was however considered acceptable with the motivation that an increased response time keeps the model conservative compared to a real-life system in which the heat transfer would be quicker.

Another considered approach was to increase the accuracy of the model by either implementing and solving the Navier-Stokes equations or by using a multi-physics solver. This would allow for the inclusion of convective heat transfer in the model, but it would simultaneously increase the complexity and the computational cost. Methods with a higher order of accuracy is intended to be implemented in future studies, but for this preliminary analysis the first order approach is considered sufficient.

\subsubsection{Relationship between temperature and inserted height}

Given that the upper reservoir, which contains the expansion liquid, is completely filled, the volumetric expansion caused by a temperature increase has to propagate downwards through the inner ARC tube and subsequently push the absorber liquid upwards into the core. Under the assumption that no volumetric expansion occurs inside the ARC tubes or in the lower reservoir, and that there is no thermal expansion of the ARC structure, it is possible to formulate a correlation that describes the inserted height of absorber liquid as a function of the mean temperature in the upper reservoir. This correlation takes as input the mean temperature in the upper reservoir, derived in the previous section, and returns the inserted height of absorber liquid. The inserted height is thereafter passed to the final part of the ARC model.

It is important to note that this method of modelling maintains a conservative approach with regards to inserted negative reactivity as a function of temperature. For instance, volumetric expansion due 
to an increase in temperature was assumed to only occur within the upper reservoir. However, in a real system there would also be volumetric expansion within the ARC tubes and in the lower reservoir. This simplification implies that in reality the absorber liquid would be inserted further into the core for a given temperature increase compared to what the model predicts.

\subsubsection{Inserted reactivity}

To conclude the ARC modelling, a correlation between the amount of inserted reactivity for a given inserted height of absorber liquid was derived. Successive simulations to obtain $k_{e f f}$ values were performed with the Monte Carlo code Serpent2 [15], in which the absorber liquid was inserted further into the core at every iteration. With this procedure a set of data points that relates $k_{\text {eff }}$ to inserted distance of absorber liquid was obtained, and Matlab's Curve Fitting Toolbox was subsequently used to fit a function to the data. A third order Fourier series turned out to yield the best fit, and the resulting function can be seen plotted along with the data points in Figure 3. A Fourier series was chosen for its good fit to the data, it does however not have any real physical interpretation in this context. Another class of functions might perhaps be a more physically correct choice when describing the S-curve in Figure 3.

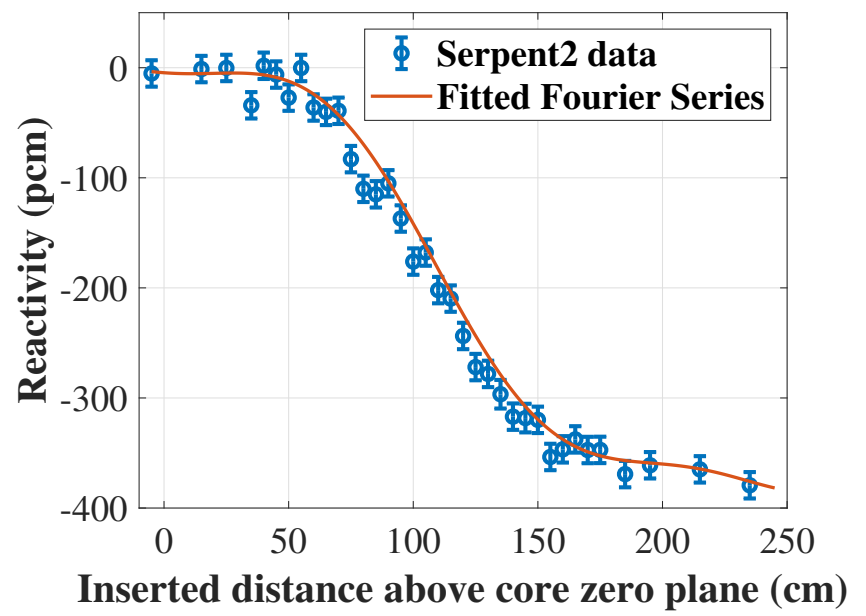

Figure 3: ARC S-curve along with fitted Fourier series function within studied interval.

Along with a model of the SEALER-UK reactor the three aforementioned sections are implemented into BELLA for dynamic simulations of an unprotected transient.

\section{RESULTS}

In this paper we investigated the impact on core safety, and more specifically core temperatures, when implementing the ARC system in a small lead-cooled fast reactor. In particular, the Unprotected Transient Overpower (UTOP) scenario, in which $350 \mathrm{pcm}$ of reactivity was inserted into the core during a time period of one (1) second, was analysed. This scenario could represent a severe control rod assembly ejection accident, in which one or two control rod assembly drive mechanisms malfunction and the assemblies are ejected from the core. 
Simulations with Serpent 2 shows that the reactivity worth of one control rod assembly is in the order of $140 \mathrm{pcm}$, and with this in mind one can argue that the investigated transient is well beyond anything reasonably expected to occur. However, it was decided to insert a value equal to the ARC system's ability to insert negative reactivity in order to study its behaviour in an extreme scenario. The result from said reactivity insertion is seen in Figure 4 hereinunder, where the two cases with and without an ARC system are investigated. Temperatures are plotted for the fuel centreline and for the coolant outlet.

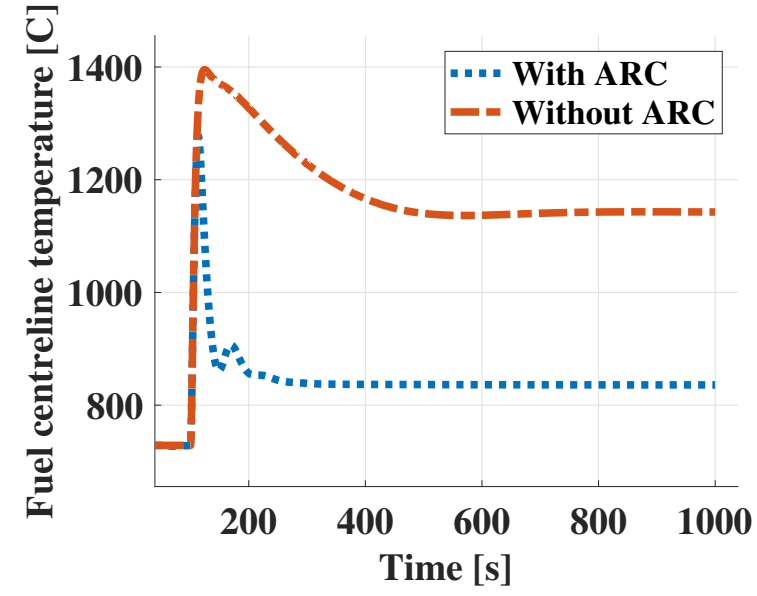

(a) Fuel Centre Line Temperature.

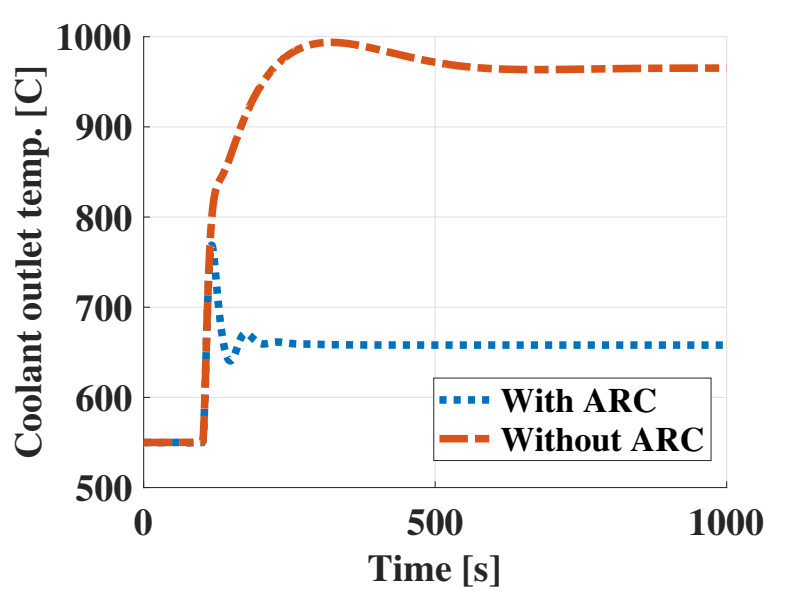

(b) Coolant Outlet Tube Temperature.

Figure 4: Temperatures during a UTOP $(350 \mathrm{pcm}$ in $1 \mathrm{~s})$, with and without the ARC system.

As seen in Figure 4, the ARC system provides a distinct and positive contribution to the safe operation of the reactor, even in the case of a transient well beyond anything foreseeable. For both temperatures shown in Figure 4, the steady state value ends up around $300 \mathrm{~K}$ lower with the ARC system deployed compared to when it is not.

\section{CONCLUSIONS}

It was shown in this paper that the ARC system forcefully mitigates a severe uncontained transient accident and that it manages to maintain the fuel centreline and coolant outlet temperatures at values which are around $300 \mathrm{~K}$ lower compared to an identical transient without using the ARC system. Many of the design choices made in this work are considered quite general and arbitrary, and a reason for this, are among other things, that the final design of the SEALER-UK reactor wasn't completed when this work was conducted.

At the time of writing this paper no verified models to describe a loss of flow or a loss of heat sink accident had been developed for the SEALER-UK reactor. This development is expected to be performed in future studies in order to verify the behaviour of the ARC system during other anticipated transients. Future research is also intended to include the implementation of the ARC system in other types of next generation reactors, such as sodium fast reactor where the ARC potentially might prevent sodium boiling by maintaining lower coolant temperatures during transients. 


\section{REFERENCES}

[1] IPCC. "Summary for Policymakers." In P. Z. V. Masson-Delmotte and H. O. Pörtner, editors, Global Warming of $1.5^{\circ} \mathrm{C}$. An IPCC Special Report on the impacts of global warming of $1.5^{\circ} \mathrm{C}$ above preindustrial levels and related global greenhouse gas emission pathways, in the context of strengthening the global response to the threat of climate change, sustainable development, and efforts to eradicate poverty. World Meteorological Organization, Geneva, Switzerland (2018).

[2] S. Qvist and E. Greenspan. "An Autonomous Reactivity Control system for improved fast reactor safety." Progress in Nuclear Energy, volume 77, pp. 32-47 (2014).

[3] S. A. Qvist, C. Hellesen, R. Thiele, A. E. Dubberley, M. Gradecka, and E. Greenspan. "Autonomous Reactivity Control (ARC) - Principles, geometry and design process." Nuclear Engineering and Design, volume 307, pp. 249-274 (2016).

[4] S. A. Qvist, C. Hellesen, M. Gradecka, A. E. Dubberley, T. H. Fanning, and E. Greenspan. "Tailoring the response of Autonomous Reactivity Control (ARC) systems." Annals of Nuclear Energy, volume 99, pp. 383-398 (2017).

[5] LeadCold Reactors. "SEALER-UK plant design submitted for review." URL https://www.leadcold. com/sealer-uk-design-submitted.html.

[6] E. Suvdantsetseg, S. Qvist, and E. Greenspan. "Preliminary transient analysis of the Autonomous Reactivity Control system for fast reactors." Annals of Nuclear Energy, volume 77, pp. 47-64 (2015).

[7] T. Lindström. SPARC fast reactor design: Design of two passively safe metal-fuelled sodium-cooled pool-type small modular fast reactors with Autonomous Reactivity Control. M.sc. thesis, Uppsala University (2015).

[8] J. Wallenius, S. Qvist, I. Mickus, S. Bortot, P. Szakálos, and J. Ejenstam. "Design of SEALER, a very small lead-cooled reactor for commercial power production in off-grid applications." Nuclear Engineering and Design, volume 338, pp. 23-33 (2018).

[9] J. Ejenstam, M. Halvarsson, J. Weidow, B. Jönsson, and P. Szakalos. "Oxidation studies of Fe10CrAl-RE alloys exposed to $\mathrm{Pb}$ at $550{ }^{\circ} \mathrm{C}$ for 10,000 h." Journal of Nuclear Materials, volume 443(1-3), pp. 161-170 (2013).

[10] S. Bortot, Cammi, Ghazy, Ricotti, Camprini, and Artioli. "Transient evaluation of a GEN-IV LFR demonstration plant through lumped parameters analysis of coupled kinetics and thermal-hydraulics." Proc. ICAPP '10: 2010 International Congress on Advances in Nuclear Power Plants (2010).

[11] S. Bortot, A. Cammi, and C. Artioli. "Dynamic performance assessment of MOX and metallic fuel core options for a Gen-IV LFR demonstrator." Progress in Nuclear Energy, volume 54(1), pp. 112122 (2012).

[12] S. Bortot, Cammi, Lorenzi, Ponciroli, D. Bona, and Juarez. "Stability analyses for the European LFR demonstrator." Nuclear Engineering and Design, volume 265, pp. 1238-1245 (2013).

[13] S. Bortot, E. Suvdantsetseg, and J. Wallenius. "BELLA: a multi-point dynamics code for safetyinformed design of fast reactors." Annals of Nuclear Energy, volume 85, pp. 228-235 (2015).

[14] J. Wallenius, S. Bortot, and I. Mickus. "Unprotected transients in SEALER: A small lead-cooled reactor for commercial power production in Arctic regions.” Proc. PHYSOR 2018 (2018).

[15] J. Leppänen, M. Pusa, T. Viitanen, V. Valtavirta, and T. Kaltiaisenaho. "The Serpent Monte Carlo code: Status, development and applications in 2013." Annals of Nuclear Energy, volume 82, pp. $142-150$ (2015). 